\title{
Scientific review of the aesthetic uses of botulinum toxin type A
}

\author{
Mee Young Park ${ }^{1}$, \\ Ki Young Ahn ${ }^{2}$ \\ ${ }^{1}$ Department of Neurology, Yeungnam \\ University Medical Center, Daegu; ${ }^{2}$ Dr. \\ Ahn's Aesthetic and Plastic Surgical \\ Clinic and Botulinum Center, Daegu, \\ Korea
}

\begin{abstract}
Botulinum toxin type A (BoNT-A), onabotulinumtoxinA (Botox) was approved by the United States Food and Drug Administration for temporary improvement of glabellar lines in patients 65 years and younger in 2002, and has also been used widely for aesthetic purposes such as hyperhidrosis, body shape contouring, and other noninvasive facial procedures. BoNT-A inhibits presynaptic exocytosis of acetylcholine (ACh)-containing vesicles into the neuromuscular junction at cholinergic nerve endings of the peripheral nervous system, thereby paralyzing skeletal muscles. ACh is the most broadly used neurotransmitter in the somatic nervous system, preganglionic and postganglionic fibers of parasympathetic nerves, and preganglionic fibers or postganglionic sudomotor nerves of sympathetic nerves. The scientific basis for using BoNT-A in various cosmetic procedures is that its function goes beyond the dual role of muscle paralysis and neuromodulation by inhibiting the secretion of ACh. Although the major target organs for aesthetic procedures are facial expression muscles, skeletal body muscles, salivary glands, and sweat glands, which are innervated by the somatic or autonomic nerves of the peripheral cholinergic nerve system, few studies have attempted to directly explain the anatomy of the areas targeted for injection by addressing the neural physiology and rationale for specific aesthetic applications of BoNT-A therapy. In this article, we classify the various cosmetic uses of BoNT-A according to the relevant component of the peripheral nervous system, and describe scientific theories regarding the anatomy and physiology of the cholinergic nervous system. We also review critical physiological factors and conditions influencing the efficacy of BoNT-A for the rational aesthetic use of BoNT-A. We hope that this comprehensive review helps promote management policies to support long-term, safe, successful practice. Furthermore, based on this, we look forward to developing and expanding new advanced indications for the aesthetic use of BoNT-A in the future.
\end{abstract}

Keywords: Acetylcholine / Botulinum toxin, type A / Cholinergic nervous system / Diffusion / Dilution / Esthetics / Motor endplate / Pharmacology

\section{INTRODUCTION}

Approximately 20 years have passed since Botox was approved by the United States Food and Drug Administration for the treatment of neurological diseases [1], and its treatment range

\section{Correspondence: Ki Young Ahn}

Dr. Ahn's Aesthetic and Plastic Surgical Clinic and Botulinum Center,

3 Dongseong-ro 11-gil, Jung-gu, Daegu 41942, Korea

E-mail: kyahn3010@naver.com

Received February 2, 2021 / Revised February 2, 2021 / Accepted February 5, 2021 has expanded beyond neuromuscular diseases to also encompass aesthetic procedures, pain conditions, and sphincter-related disorders. Theoretically, botulinum toxins (BoNTs) weaken skeletal muscles by presynaptic blockade of the exocytosis of acetylcholine (ACh)-containing vesicles in the neuromuscular junction and eliminate facial wrinkles. In addition to the elimination of facial wrinkles, the cosmetic uses of botulinum toxin type A (BoNT-A) include contouring of the lower face, neckline, and lower leg. It is also used to treat local hyperhidrosis of 
the axilla, forehead, palm, and scalp. Moreover, it is used for the management of gummy smile, facial asymmetry, and skin rejuvenation. Recently, novel attempts have been made to contour the lower face by using BoNTs to cause atrophy of the salivary glands. The various clinical therapeutic effects of BoNTs arise from simply blocking the secretion of $\mathrm{ACh}$, which is the most broadly used neurotransmitter in the somatic nervous system, preganglionic and postganglionic fibers of parasympathetic nerves, and preganglionic fibers and some postganglionic fibers of sympathetic nerves. ACh is also used as a neurotransmitter instead of norepinephrine in some postganglionic fibers of sympathetic nerves involved in secretions from sweat glands. Some postganglionic fibers of parasympathetic nerves are involved in secretions from the salivary glands. ACh plays a dual role, acting directly on the target organ and modulating neuroactive amino acids, and this dual role has led to an expansion of its clinical indications. For instance, it is used to prevent chronic migraine $[2,3]$ and treat neuralgia or peripheral neuropathic pain [4]. Although the current theoretical basis is insufficient, there are certain medical conditions for which BoNT-A treatment, such as mesobotox, is effective [5]. However, the theoretical basis can be deduced through an anatomical and pathological analysis of the disease and consideration of the relevant mechanism of action of BoNT-A.

A comprehensive understanding of cholinergic nervous system-related aesthetic indications of BoNT-A, in combination with associated physiological factors and conditions such as dosing, volume and dilution, distribution characteristics, and injection techniques influencing the efficacy of BoNT-A, can promote more sophisticated treatment techniques and management policies to obtain the best efficacy while minimizing side effects, thereby helping to expand the new advanced clinical indications for the cosmetic use of BoNT-A.

\section{BOTULINUM NEUROTOXIN: A MIRACLE POISON}

BoNT-A inhibits the secretion of the neurotransmitter ACh at cholinergic nerve endings in the peripheral nervous system (PNS).

A cholinergic neuron is a nerve cell that mainly uses the neurotransmitter ACh to send its messages to target organs. The development of a BoNT-A treatment, the first microbial toxin treatment used in humans, began with the pathophysiological hypothesis that toxins extracted from spoiled sausage interfere with signaling in the motor nervous system in a book published by Justinus Wurst Kerner in 1822 [6]. Then, Burgen et al. [7] found that BoNT interferes with muscle nerve transmission in
1949. In 1954, Brooks [8] published a paper stating that the injection of BoNT into a hyperactive muscle prevents ACh release from motor nerve endings. These hypothesis-confirmation studies were published over a period of roughly 100 years. Thereafter, another 30 years passed before BoNT was developed as a human treatment, when Scott [9] published a paper on the results of using Oculinum, a commercial product of BoNT-A, in 1980. In 1989, BoNT-A was approved for the nonsurgical correction of strabismus, blepharospasm, hemifacial spasm, and Meige syndrome in adults. Shortly thereafter, Allergan bought Scott Company and renamed the toxin. Botox was born [10].

In 1987, the ophthalmologist Jean Carruthers discovered the effect of Botox on facial rhytides when a patient she treated for blepharospasm requested to have her forehead injected because of the improvement she observed in her periorbital region. Eventually, in 1992, Alastair Carruthers (her spouse, a dermatologist) published a paper reporting that Botox injection removed glabellar wrinkles, which became the legendary beginning of cosmetic treatment using BoNTs [11]. Similarly, in 2000, the Korean plastic surgeon KY Ahn published the first paper in Asia on the long-term follow-up effect of Botox injections for the treatment of crows' feet and forehead wrinkles together with MY Park (his spouse, a neurologist) [12]. It has now become the most common cosmetic procedure in the United States [13].

\section{CHOLINERGIC NERVOUS SYSTEM}

The nervous system of our body is largely divided into the central nervous system (CNS) and the PNS. The CNS consists of the brain and spinal cord, and the PNS is divided into the somatic nervous system and the autonomic nervous system (ANS). The peripheral somatic nervous system can be divided into cranial nerves (CNs), motor nerves, and sensory nerves, and causes muscles to move according to stimuli received from the CNS or transmits the stimuli felt by the sensory organs to the CNS. The ANS, which is responsible for involuntary functions, such as digestion, breathing, heart beating, and sweat secretion, can be divided into sympathetic nerves and parasympathetic nerves. The sympathetic and parasympathetic nerves maintain a balance so that the homeostasis of various organs is not biased to one side. The somatic nervous system, preganglionic and postganglionic fibers of parasympathetic nerves, and preganglionic fibers and some postganglionic fibers of sympathetic nerves contain cholinergic neurons. Cholinergic neurons mainly use $\mathrm{ACh}$ as a neurotransmitter to send messages. $\mathrm{ACh}$ is the most broadly used neurotransmitter, and functions in 
both the CNS and the PNS. In the CNS, cholinergic projections from the basal forebrain to the cerebral cortex and hippocampus support the cognitive functions of those target areas. In the PNS, ACh activates skeletal muscles in the somatic nervous system, and ACh is also a major neurotransmitter in the ANS. Projections from the CNS to the autonomic ganglia use $\mathrm{ACh}$ as a neurotransmitter to excite ganglionic neurons. In parasympathetic nervous system output connections, the projections from ganglionic neurons to target organs also release $\mathrm{ACh}$, but act on muscarinic receptors and induce vasodilation. In the sympathetic nervous system, the output connections mainly release noradrenaline, although ACh is released at a few points, such as the sudomotor innervation of the sweat glands. Some postganglionic fibers of parasympathetic nerves are involved in secretions from the salivary glands [14].

Advances in understanding postsynaptic receptors have recently increased at a rapid pace, owing largely to studies of the $\mathrm{ACh}$ receptor $(\mathrm{AChR})$ at the motor endplate. The endplate AChR belongs to a large superfamily of neurotransmitter receptors, called Cys-loop receptors, and has served as an exemplar receptor for probing fundamental structures and mechanisms that underlie fast synaptic transmission in the CNS and PNS [15]. Recent studies have provided an increasingly detailed picture of the structure of the AChR and the symphony of molecular motions that underpin its remarkably fast and efficient chemo-electrical transduction.

\section{Acetylcholine, a neuro-modulatory neurotransmitter}

ACh is composed of an ester of acetic acid and choline, and is synthesized from choline and acetyl-CoA by choline acetyltransferase in cholinergic neurons. When nervous stimuli reach the cholinergic nerve ending, ACh is secreted and neurotransmission occurs. ACh is the chemical that motor neurons of the nervous system release to activate muscles. It is also a neurotransmitter in the ANS, both as an internal transmitter for the sympathetic nervous system and as the final product released by the parasympathetic nervous system, where it serves as the primary neurotransmitter.

\section{Structure and action mechanism of BoNT-A}

BoNT-A is produced by Clostridium botulinum, a Gram-positive, rod-shaped anaerobic bacterium and has a molecular weight (MW) of about $150 \mathrm{kDa}$, which is composed of singlechained polypeptides. It consists of a heavy chain $(\mathrm{Hc})$ of 100 $\mathrm{kDa}$ and a light chain $(\mathrm{Lc})$ of $50 \mathrm{kDa}$, and these two chains are connected by a disulfide bond. According to the serotype, BoNT can be divided into seven serotypes: BoNT A, B, C(1,2), D, E, F, and $G$ [16]. The human nervous system is susceptible to five toxin serotypes (BoNT-A, B, E, F, G) and unaffected by two (BoNT-C, D). Among those, BoNT-A is the most potent, but BoNT-A and BoNT-B are approved for clinical use only. The action mechanism of BoNT-A is largely divided into four stages: (1) binding, (2) internalization, (3) disulfide reduction and translocation, and (4) inhibition of neuromuscular release (Fig. 1) [17]. Like most bacterial toxins, BoNT is internalized by receptor-mediated endocytosis. In other words, BoNT is internalized through a receptor in the nerve ending of the cholinergic neuron. To obtain chemical efficacy, the single disulfide bond between the $\mathrm{Hc}$ and Lc must be well maintained until the intracellular internalization of BoNT-A. The C-terminal of the 100$\mathrm{kDa} \mathrm{Hc}$ of BoNT-A enables BoNT-A to bind to the presynaptic cholinergic receptor, in a process called internalization. Once internalized, the disulfide bond that binds $\mathrm{Hc}$ and Lc is cleaved, and the reduced Lc is then transported across the vesicular membrane into the neuronal cytosol through the ATP-dependent receptor-mediated endocytotic pathway, in a process known as translocation. Finally, the reduced Lc of BoNT-A can have an intracellular toxic moiety and eventually inhibits ACh exocytosis (inhibition of neuromuscular release) by blocking SNAP-25, a SNARE protein. The N-terminal of Lc is a zinc-dependent protease that cleaves one or more of the SNARE complexes [18]. Internalization is maximal (in vitro at least) after about 90 minutes at $22^{\circ} \mathrm{C}$ [19].

Because the disulfide bond is very weak [20], careful attention is required to obtain maximal efficacy at the same dose when saline reconstitution is used. When reconstituting BoNT-A with normal saline, one should not shake the vial vigorously. The disulfide bond is so fragile that even a massage immediately after injection should be avoided; it should also be noted that massage immediately after the procedure may sometimes cause bruising at the injection site as well.

SNAREs complex, the membrane fusion proteins transport ACh vesicles from the cytosol to the synaptic cleft

Normally, to release ACh from the presynaptic vesicle of the cholinergic nerve terminal, soluble NSF (N-ethylmaleimidesensitive factor) attachment protein receptor (SNARE) proteins are necessary. These are membrane fusion proteins where synaptic vesicle release occurs. The SNARE complex consists of five vesicle conjugation factors that move $\mathrm{ACh}$ vesicles in the cytosol to the cytoplasmic membrane. In the SNARE complex, cytoplasmic proteins include a-SNAP (soluble N-ethylmaleimide attachment protein), $\gamma$-SNAP (synaptosomal-associated protein), NSF (N-ethylmaleimide-sensitive fusion protein), and SNAP-25 (SNAP-25kDa); vesicle proteins include VAMP (vesi- 


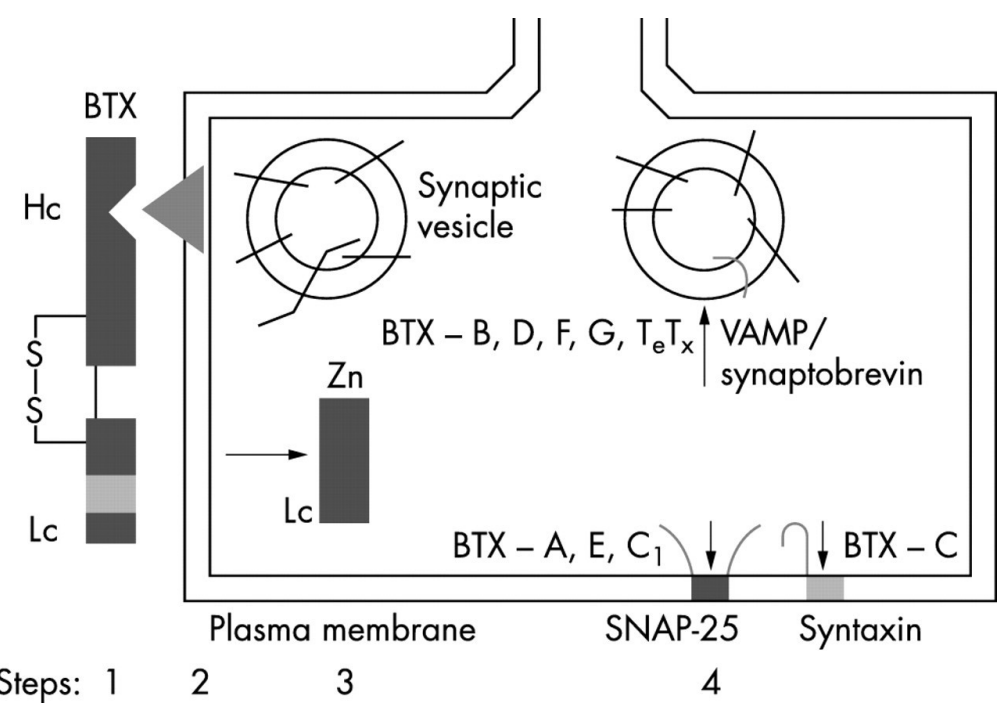

Fig. 1. Mechanism of action of botulinum toxin (a four-step process). Step 1: after botulinum toxin is activated by proteolytic cleavage of the polypeptide chain into a $100 \mathrm{kDa}$ heavy chain $(\mathrm{Hc})$ and a $50 \mathrm{kDa}$ light chain $(\mathrm{Lc})$, linked by a disulphide bond (S-S); the Hc domain of the toxin binds to the presynaptic plasma membrane of the motor axon terminal. Step 2: the toxin complex is then internalized by energy-dependent endocytosis. Step 3: the Lc, a zinc endopeptidase, is released into the cytoplasm. Step 4: the Lc cleaves various components of SNARE (indicated by vertical arrows), including SNAP 25 (botulinum toxin A), VAMP/synaptobrevin (botulinum toxin B), or syntaxin (botulinum toxin C), and thus prevents the fusion of the acetylcholine synaptic vesicle with the plasma membrane. This blocks the release of the neurotransmitter into the synaptic cleft, causing local chemodenervation. SNARE, soluble NSF (N-ethylmaleimide-sensitive factor) attachment protein receptor; SNAP, synaptosome associated protein; VAMP, vesicle-associated membrane protein; BTX, botulinum toxin. Reprinted from Jankovic. J Neurol Neurosurg Psychiatry 2004;75:951-7 [17].

cle-associated membrane protein)/synaptobrevin; and the target membrane protein is syntaxin. Among these proteins, the BoNT-A type inhibits the exocytosis of ACh by inactivating SNAP-25, one of several vesicle conjugation factors. In addition, BoNT-E and BoNT-C also inactivate SNAP-25; BoNT-B, BoNT-D, BoNT-F, and BoNT-G inactivate VAMP/synaptobrevin, and BoNT-C also inactivates syntaxin, thereby interfering with the secretion of ACh from synaptic vesicles. When this transport is interrupted, the release of $\mathrm{ACh}$ from cholinergic nerve endings is blocked, resulting in muscle paresis or reduction of glandular secretions [21].

This denervation mechanism of BoNT-A is reversible. New axons sprout from the terminal axons of the unmyelinated motor nerve or from Ranvier's nodes of myelinated motor nerves. Axonal arborization causes axon branching, leading to recovery of the function of the paralyzed muscle. However, it was originally believed that the cholinergic blockade of a specific nerve terminal was permanent and that reinnervation of the target tissue was caused by axonal resprouting. However, animal data with BoNT-A has demonstrated that sprouting produces temporary reinnervation only during the early recovery phase. During the late recovery phase, the sprouts retract and the original terminal becomes functional once again. After reinnervation is completed, the target tissue becomes fully functional [22].

\section{THE CLASSIFICATION OF AESTHETIC INDICATIONS ACCORDING TO THE TARGETED NERVOUS SYSTEM}

Since Carruthers and Carruthers [11] published the world's first paper on the effects of BoNT-A in the treatment of glabellar wrinkles in 1992, BoNT-A has been most widely used for cosmetic purposes in various areas of the human body, such as facial wrinkle removal and lower face, calf, and neckline contouring [13]. In addition, BoNT-A is used to treat focal hyperhidrosis in the axilla, forehead, hand, and scalp. It has recently been used for facial contouring that combined facial liposuction and facelift by using submandibular salivary gland atrophy as an adverse effect of BoNT-A [23]. We classify these various cosmetic uses according to the involved nervous system to expand the therapeutic indications of BoNT by knowing basic anatomy.

In the $\mathrm{CN}$ or the peripheral motor nerve of the somatic nervous system, BoNT-A is used for eliminating habitual or hyperactive facial wrinkles by injecting it into the facial expression muscles that are supplied by the somatic motor nerve of the facial nerve (seventh CN) [11,12]. According to our clinical experience, long-term repeated injections of BoNT-A into slightly 
larger muscles lead to muscular atrophy and volume loss around the injected muscle [24,25]. Facial expression muscles are thin and atrophy is difficult to notice, but atrophy is easily observed in rather thick muscles such as the masseter, trapezius, and calf muscles. Therefore, by using the concept of muscle atrophy resulting from the side effects of BoNT-A treatment, noninvasive correction of pathological muscle hypertrophy and cosmetic contouring are possible. Specifically, these procedures include lower face contouring for masseter hypertrophy [24,25], via injection into the masseter innervated by the anterior division of the mandibular division of the trigeminal nerve (fifth $\mathrm{CN}$ ), and lower leg contouring by injection into the calf muscles [26], which are innervated by the tibial nerve, which eventually separates from the sciatic nerve. Slim and elegant neck and shoulder line contouring is being attempted by using motor function paralysis of the trapezius, which is supplied directly from the accessory nerve (11th CN) [27]. Two months after the injection of 10 points, a total of $50 \mathrm{U}$ of BoNT-A into each side of the trapezius, the neck-shoulder angle widened from $116^{\circ}$ to $122^{\circ}$ in our personal representative case of contouring the shoulder line.

Recently, the cosmetic use of salivary gland atrophy by parasympathetic nerve blocking has also been tried in combination with facial contouring procedures such as facelift or liposuction in Korea. These procedures include lower face or mandibular line contouring by BoNT injection into the parotid gland or submandibular gland. The parotid gland receives both sensory and autonomic innervation. The autonomic innervation controls the rate of saliva production and is supplied by the glossopharyngeal nerve (ninth $\mathrm{CN}$ ). The glossopharyngeal nerve synapses with the otic ganglion, and then postganglionic parasympathetic fibers innervate the auriculotemporal nerve, a branch of the mandibular nerve. The submandibular glands receive their parasympathetic input via the chorda tympani nerve, which is a branch of the facial nerve (seventh $\mathrm{CN}$ ) via the submandibular ganglion. The postganglionic fibers reach the submandibular gland and release ACh. The nerve function is a secretomotor capacity. The sudomotor nerve to the sweat glands is supplied by some postganglionic nerves of the sympathetic nerve. The postganglionic neuron for sweat gland innervation differs from other sympathetic postganglionic neurons in that it releases ACh to act on muscarinic receptors. It is used for the treatment of focal hyperhidrosis in the axilla, hand palm, forehead, and scalp by intradermal injection into the eccrine sweat gland in the dermis with BoNT-A. A gingival smile (or gummy smile) is due to an excessive vertical maxilla, hyperfunction of the lip elevator, and delayed passive eruption. Injection of only a small dose of BoNT-A (2.5 units per $0.1 \mathrm{~mL}$ on each side) $3 \mathrm{~mm}$ lateral to the alar-fascial groove at the level of the nostril opening at the insertion of the levator labii superioris alaeque nasi muscle improved gummy smile in the gingival display [28]. This procedure is a simple and good example of obtaining a successful result and minimizing side effects by analyzing the dynamic physiology of upper lip anatomy. Another useful treatment example is that BoNT injections in the platysma muscle will produce relaxation of the platysma bands, improve the mandibular line, and lessen the marionette line by reducing the tension of the platysma, together with the depressor auricular oris (DAO) muscle, which pulls the buccal commissures laterally downward, and decrease the horizontal neck lines [29]. The main connections of the platysma muscle are (1) superior and medially, with the lateral part of the orbicularis oris muscle, and (2) medially in the mandibular region, the anterosuperior fibers blend with the posterior border of the DAO muscle. Therefore, would not these injections be killing two birds with one stone?

A systematic review and meta-analysis showed that injections of intralesional BoNT-A were more effective in the treatment of hypertrophic scars and keloids than injections of intralesional corticosteroid or placebo [30]. The proposed rationale for scar treatment using BoNT-A involves not only chemical immobilization of the muscles around the scar, but also direct modulation of the activity of fibroblasts by altering apoptotic, migratory, and fibrotic pathways. BoNT-A has the potential to reduce the tension produced during the wound healing process by paralyzing adjacent muscles. Moreover, BoNT-A can attenuate the inflammatory response related to cellular metabolic activity, which is believed to support scar hypertrophy. Several studies have demonstrated that BoNT-A plays the following roles in regulating fibroblast activity: (1) decreasing transforming growth factor- $\beta 1$ and connective tissue growth factor, which inhibit fibroblast proliferation; (2) regulating the fibroblast cell cycle; and (3) preventing differentiation from fibroblasts to myofibroblasts. However, more evidence-based research is needed to elucidate the role of BoNTs in scar treatment or keloid scar management protocols.

Finally, it is worthwhile to mention the use of microdroplets of BoNT-A, also called mesobotox or microbotox, which is the injection maneuver of multiple microinjections of a diluted BoNT-A into the dermis or interface between the dermis and the superficial layer of the facial muscles, glands, and skin structures. The mesobotox procedure is mesotherapy using BoNT as a mesoproduct [5,31]. This emerging technique is considered to be a possible approach for BoNT treatment for facial rejuvenation temporally. However, the scientific basis of mesobotox procedures for facial complexion improvement re- 
mains insufficient. The proposed hypotheses regarding the effects of mesobotox are mainly due to slight muscle paralysis resulting in (1) a facial lifting effect by slight paralysis of the depressor muscles (e.g., the platysma); (2) reducing wrinkles by mild paralysis of the facial expression muscles; and (3) smoothing and tightening the skin texture by paralysis of arrector pili muscles to reduce pore size and inducing partial lymphatic drainage insufficiency to make the face look plump. In addition, inhibition of exocrine function results in (1) a clean face by reducing the acne and pore size due to decrease in sebum and sweat production by inhibition of $\mathrm{ACh}$ and substance $\mathrm{P}$ release; and (2) whitening of the face by inhibition of the release of parasympathetic-innervating perivascular neuropeptides, such as calcitonin gene-related peptide (a potent general arterial vasodilator) and vasoactive intestinal peptide, to reduce facial flushing. Although the mesobotox procedure is performed with the desire to have a well-hydrated, shiny appearance and younger face, there are limitations such as short effective duration and disadvantages in terms of immunological safety if the procedure is repeated within 3 months. Overall, more scientific evidence is needed.

\section{TARGETING THE NEUROMUSCULAR JUNCTION}

The anatomy of the target organ is complex as the neighboring muscles are close to each other, interacting and interfacing at different levels and depths to execute heterogeneous functions. Knowledge of the relevant anatomy and the relationships of this three-dimensional anatomical configuration makes it possible to determine the exact muscle for injections to achieve successful therapeutic outcomes without adverse effects. Once the muscle to inject is decided, the injection should be aimed at the motor endplate zones to obtain the maximal effect of BoNT-A in the target skeletal muscle. Because the location of the neuromuscular junction (found in motor endplates where many toxin receptors for internalization are scattered) is most highly concentrated in a band within the mid-belly of the muscle, injections of botulinum neurotoxins are hypothesized to be more potent when specifically targeted toward the motor endplate zones [32]. Motor points (the area in which a minimal-intensity, short-duration electrical stimulus will cause a muscle contraction, usually corresponding anatomically to the location of the motor endplate zones) are probably reasonably close and may be a good alternative in some situations in which small motor nerve endings enter into a portion of the muscle rather than a motor endplate zone in which ACh is released. Clinically, such a motor endplate zone is in the area where strong mus- cle tone is felt by palpation or the area where the muscle is hypertrophied remarkably.

However, since motor endplate zones do not necessarily coincide with the motor points, there is some difficulty in clinical practice. Electromyography, or electrical stimulation, and ultrasound guidance is required for localization of these zones. Motor endplates are not randomly scattered throughout skeletal muscle, but occur in groups or bands in most cases. The distribution of these locations is relatively well described in humans and coincides with motor point maps. The use of electromyography to localize BoNT-A injections at (or very near to) motor endplate zones potentiate the paralytic effects of BoNT-A. Furthermore, the most effective paralysis with minimal side effects might be obtained by multiple injections subdividing the whole amount of BoNT-A along the motor endplates' bend, rather than a single bolus injection in the target muscle, by lessening the spread of toxin into neighboring muscles through the fascia. These factors are especially important when performing a procedure to create smooth lines and contours without impairing the inherent function of the muscles, such as in the lower face or calf muscle.

\section{FACTORS INFLUENCING EFFICACY AND SAFETY}

\section{Diffusion, spread, and migration of BoNT-A}

Besides accurate selection and identification of intended targets, the local and systemic distribution of BoNT-A has a significant impact on efficacy and safety. The following concepts should be carefully distinguished: (1) diffusion refers to a more microscopic phenomenon in which a soluble molecule is dispersed by passive transport beyond its original injection site; (2) spread refers to physical movement of the toxin from one site to another, and it depends on many variables related to the injection technique, volume, needle size, and other physical factors; (3) migration refers to spread to distant sites, and can occur either via nerves (neuroaxonal transport) or by blood (hematogenous transport); and (4) volume and dilution [33]. These concepts are very important in neurotoxin treatment because they are largely responsible for significant side effects. As an extreme example, patients with neuromuscular disorders (e.g., myasthenia gravis, amyotrophic lateral sclerosis) or incapacitated patients (e.g., cerebrovascular accident) who are injected with a large amount of BoNT-A may experience further compromise of their respiratory function as a result of the spread of the biological activity of BoNT-A from the injection site into the respiratory muscles.

Hypothetically, diffusion of the neurotoxin into adjacent tis- 
sues may be slower for a high-molecular-weight complex than for a lower-molecular-weight complex or free neurotoxin. However, this possibility is unclear because the degree of diffusion of three different commercial preparations of BoNT-A, onabotulinumtoxinA (Botox; $900 \mathrm{kDa} \mathrm{MW}$ ), abobotulinumtoxinA (Dysport; 500-900 kDa MW), and incobotulinumtoxi$\mathrm{nA}$ (Xeomin; $150 \mathrm{kDa} \mathrm{MW}$ ) was similar in a mouse study [34]. Those researchers found that the highest level of neural cell adhesion molecule (N-CAM) expression (which has been used as an index of diffusion because it can be detected in regenerated adult muscle in BoNT-A treated muscles) was found in the injection site, whereas only a limited amount of N-CAM expression is found in adjacent muscles. However, another study using RT002 (composed of a 150kDa purified BoNT-A) showed significantly less diffusion, 58\%-100\% longer lasting muscle force generation inhibition, and 100\%-126\% longer duration of the therapeutic effect in RT002, while causing an equivalent or lesser degree of systemic side effects in mice compared with onabotulinumtoxinA (Botox) [35]. Thus, additional research is needed on product-specific differences.

The spread of BoNT-A is most likely caused by the diffusion of unbound toxin through the extracellular space, driven by the concentration gradient and the dynamics of the injection. Dose-dependent and passive kinetic dispersion of the toxin away from the injection site in a gradient-dependent manner may play a role in toxin spread. Borodic et al. [36] reported that BoNT-A diffused up to $45 \mathrm{~mm}$ from the site of a single injection (10 U/0.1 $\mathrm{mL}$ injected into the rabbit longissimus dorsi). Although at lower doses $(1 \mathrm{U} / 0.1 \mathrm{~mL})$, the collapse of the diffusion gradient occurred over a $15-\mathrm{mm}$ to $30-\mathrm{mm}$ segment of muscle, at higher doses (5-10 U/0.1 mL) diffusion of the BoNT-A effect occurred throughout the entire muscle with no apparent endpoint.

The migration, which refers to the distal effects of BoNT that occur either via the nerves or by blood, is another emerging concept. BoNT can be transported to the cell soma and is released in intact form to second-order neurons via transcytosis. This cell-to-cell trafficking may allow BoNT undergoes anterograde and retrograde axonal transport to exert distant effects of toxin [37].

Although the action of BoNT in the PNS has been extensively documented, much less is known about the action of BoNT on the CNS. Although BoNT probably does not cross the bloodbrain barrier, the potential for CNS adverse effects should not be ignored in immunocompromised patients (BoNT-A, B, and E have been studied in the CNS). BoNT-A intramuscular injections have a dual effect, by directly or indirectly affecting the CNS as well as inducing paralysis of the corresponding muscle.
As evidence, it is effective in the treatment of chronic migraine and neuropathic pain syndrome beyond muscular origin pain [2-4]. Unequivocal evidence has been found that BoNT-A undergoes limited retrograde axonal transport. Studies in humans, nevertheless, support the idea that BoNT injected at therapeutic doses induces distal spinal and cortical effects. Peripherally, the injection of BoNT-A into a muscle reduces $\alpha$-motoneuron activity in extrafusal muscle fibers. Muscle spindles are simultaneously inhibited by the toxin's blockade of the $\gamma$-motoneuron control of intrafusal fibers and by its subsequent reduction of Ia afferent signaling, thereby reducing feedback to the a-motoneurons and other pathways to reduce muscle contraction [38]. The remote spread in which the effects of BoNT are observed at a considerable distance from the injection site cannot be fully explained by diffusion. Theoretically, these remote effects may depend either on the direct action of the toxin transported via the hematogenous route or on retrograde axonal transport. Indirectly, the effects of BoNT on the CNS may occur due to central reorganization [33].

In vitro studies have demonstrated that BoNT can affect the release of several neurotransmitters from the brain and spinal cord neurons. BoNT therapy also leads to altered afferent input to the CNS produced by the effect of muscle spindles. Therefore, the proposed mechanism of prevention of chronic migraine and neuropathic pain management by BoNT-A is decreasing sensitized nociception (1) by inhibiting glutamate release in peripheral tissues, (2) decreasing calcitonin gene-related peptide release in peripheral tissue, (3) decreasing transient receptor potential cation channel subfamily $\mathrm{V}$ member 1 trafficking to the peripheral neuron cell membrane, and (4) decreasing substance $\mathrm{P}$ release in peripheral tissues [39]. Furthermore, some data have demonstrated bilateral antinociceptive effects of BoNT-A even after unilateral peripheral injection.

Several pieces of evidence have demonstrated a dose-dependent effect on neuromuscular transmission in distal muscles and the CNS, possibly owing to the hematogenous spread of BoNT-A through the bloodstream. Flu-like symptoms and three reported cases of encephalitis are a possible sign of hematogenous spread or systemic immune response. In addition, the locally injected intramuscular BoNT effect on the ANS accumulates over time and leads to the development of increased residual urine volume and decreased heart rate variability in patients injected with therapeutic doses of BoNT-A and BoNTB. It has been found that the systemic spread of BoNT-B is higher and that autonomic adverse effects occur frequently, even when low or intermediate doses of this toxin are used [40]. The difference in the products' properties also may affect their local migration from the target muscle area and potency char- 
acteristics, which, in turn, may influence their efficacy, safety profile, and antigenic potential.

It is considered to be more effective to inject one target muscle evenly with several divided injection points. Therefore, the concept of toxin spreading is very important in BoNT-A injection technique, and the injection power and injection speed are also crucial factors. In other words, it could be said that the good skill in the toxin injection technique is achieved at the operator's fingertips. A slower injection with the feeling that the toxin is injected and sprayed onto the motor points at many neuromuscular junction synaptic areas will reduce its spread to the surrounding muscles and make the effect and effective duration stronger and longer, respectively. In addition, the fact that antibody formation to neurotoxins can be reduced by minimizing inactive toxins that do not internalize is considered to be as important as differences between-products. Furthermore, for botulinum injections, the depth of the injection pointer is determined according to the depth of the target tissue, and the dilution dose and the total injection dose are determined according to the size of the target tissue. The injection power and speed are important for accurate spread of the toxin to the target tissue.

\section{Volume and dilution}

In addition to the molecular size and the properties of the BoNT products, other factors such as volume and dilution influence the diffusion and spread of BoNT. The volume of solution used to inject equivalent units of BoNT also affects potency. However, there is little evidence that varying dilutions result in predictable diffusion, and whether the volume of dilution has clinically relevant implications for the potency of BoNT also has not yet been definitively proven.

The concept is generally accepted that the most effective paralysis can be obtained by delivering the toxin in highly-concentrated small volumes near the motor endplate region band of a muscle, and the effect is augmented by stretching exercises and electrical stimulation after BoNT-A injection. However, the size of the denervation field is mostly determined by the dose and volume in the targeted muscle. Therefore, injections divided into multiple points along the targeted muscle, rather than a single bolus injection, might contain biological activity within the targeted muscle. When a single bolus is injected into the center of the muscle, BoNT may spread beyond the boundaries of the intended muscle. A higher volume of lower-concentration BoNT-A results in greater diffusion and a larger affected area, with more spread of activity found in a human skin model.

Other differences may be linked to serotype-specific variability and organ-specific affinity. For example, BoNT-B seems to have more affinity for the autonomic system, as shown by its tendency to lead to less saliva production and greater constipation than BoNT-A [41].

\section{Drugs and diseases}

Several drugs that act on the neuromuscular junction interfere with or enhance the effectiveness of BoNT. BoNT use is contraindicated in patients with disorders of neuromuscular transmission (e.g., myasthenia gravis, Lambert-Eaton syndrome) [42].

\section{CONCLUSION}

Cholinergic nervous system-based BoNT injections for aesthetic purposes yield cost-effective results, and opportunities exist to expand its treatment indications. BoNT acts on cholinergic neurons-that is, it inhibits the secretion of the neurotransmitter ACh from somatic and parasympathetic nerves, as well as some sympathetic nerves. In somatic nerves, the effects of muscle paralysis and atrophy by BoNT injections are used cosmetically to treat facial wrinkles with skin rejuvenation and to contour the lower face, calf, and neck-shoulder line. In the parasympathetic nervous system, BoNTs are also used to contour the lower face and mandibular lines by using atrophy of the salivary glands. BoNT is also used to treat local hyperhidrosis in the axilla, palm, forehead, and scalp by inhibiting the release of ACh from sudomotor nerves (cholinergic innervation of the sympathetic nervous system, prominent in sweat glands) in some sympathetic nerves.

The clinical outcomes after BoNT injections depend not only on the skills of the injector, but also on the properties of commercial products and their propensity to diffuse, spread, and migrate, including volume and dilution, which may affect potency and systemic distribution to the CNS. For long-term safety in cosmetic uses of BoNT-A, care should be taken regarding denervation and atrophy of target organs caused by long-term repeated injections. For example, if prolonged atrophy of masseter can cause compensatory hypertrophy of other adjacent muscles, it could lead to temporomandibular joint disorders. As salivary glands may atrophy with age, treatment should consider functional aspects (i.e., digestion). A more thorough pre-examination considering these functional disturbances should be performed in each case. Furthermore, we should follow a treatment policy because none of the possible signs of hematogenous spread, a systemic immune response, or mild botulism after BoNT-A injection (usually dose-dependent) were noticed by patients or were clinically relevant. Flexible BoNT preparation for individual aesthetic purposes and accurate injection into the motor endplate of the target organ is the key to the 
BoNT injection method. The force and speed of injection must be considered as well.

Currently, cosmetic procedures such as mesobotox, which can apply for the Asian women whose aging related mid-face skin elasticity decreases [43], keloid or hypertrophic scar treatment, facial asymmetry correction in chronic facial palsy patients, and ptotic breast correction are being attempted with BoNT-A. However, further research is required to provide evidence supporting these challenging procedures.

\section{NOTES}

\section{Conflict of interest}

No potential conflict of interest relevant to this article was reported.

\section{ORCID}

Mee Young Park https://orcid.org/0000-0002-5683-1634 Ki Young Ahn https://orcid.org/0000-0002-6527-0277

\section{Author contribution}

Conceptualization: MYP, KYA. Methodology: MYP, KYA. Writing - original draft: MYP. Writing - review and editing: KYA. Approval of final manuscript: all authors.

\section{REFERENCES}

1. Botox (OnabotulinumtoxinA) [package insert]. Irvine (Botox) [package insert]. Irvine: Allergan; 2011.

2. Aurora SK, Dodick DW, Turkel CC, DeGryse RE, Silberstein $\mathrm{SD}$, Lipton RB, et al. OnabotulinumtoxinA for treatment of chronic migraine: results from the double-blind, randomized, placebo-controlled phase of the PREEMPT 1 trial. Cephalalgia 2010;30:793-803.

3. Diener HC, Dodick DW, Aurora SK, Turkel CC, DeGryse RE, Lipton RB, et al. OnabotulinumtoxinA for treatment of chronic migraine: results from the double-blind, randomized, placebocontrolled phase of the PREEMPT 2 trial. Cephalalgia 2010;30: 804-14

4. Wei J, Zhu X, Yang G, Shen J, Xie P, Zuo X, et al. The efficacy and safety of botulinum toxin type $\mathrm{A}$ in treatment of trigeminal neuralgia and peripheral neuropathic pain: a meta-analysis of randomized controlled trials. Brain Behav 2019;9:e01409.

5. Calvani F, Santini S, Bartoletti E, Alhadeff A. Personal technique of microinfiltration with botulin toxin: the SINB technique (superficial injection needling botulinum). Plast Surg (Oakv) 2019;27:156-61.

6. Carruthers A, Carruthers J. History of botulinum toxin for medical and aesthetic use. In: Cohen JL, Ozog DM, Porto DA, editors. Botulinum toxins: cosmetic and clinical applications. Hoboken: John Wiley \& Sons Inc.; 2017. p. 1-12.

7. Burgen AS, Dickens F, Zatman LJ. The action of botulinum toxin on the neuro-muscular junction. J Physiol 1949;109:1024.

8. Brooks VB. The action of botulinum toxin on motor-nerve filaments. J Physiol 1954;123:501-15.

9. Scott AB. Botulinum toxin injection into extraocular muscles as an alternative to strabismus surgery. Ophthalmology 1980; 87:1044-9.

10. Lipham WJ. A brief history of the clinical applications of botulinum toxin. In: Lipham WJ, editor. Cosmetic and clinical applications of botulinum toxin. Thorofare: SLACK; 2004. p. 1-3.

11. Carruthers JD, Carruthers JA. Treatment of glabellar frown lines with C. botulinum: a exotoxin. J Dermatol Surg Oncol 1992;18:17-21.

12. Ahn KY, Park MY, Park DH, Han DG. Botulinum toxin A for the treatment of facial hyperkinetic wrinkle lines in Koreans. Plast Reconstr Surg 2000;105:778-84.

13. Kane M, Donofrio L, Ascher B, Hexsel D, Monheit G, Rzany B, et al. Expanding the use of neurotoxins in facial aesthetics: a consensus panel's assessment and recommendations. J Drugs Dermatol 2010;9(1 Suppl):s7-22.

14. Ahmed NY, Knowles R, Dehorter N. New insights into cholinergic neuron diversity. Front Mol Neurosci 2019;12:204.

15. Sine SM. End-plate acetylcholine receptor: structure, mechanism, pharmacology, and disease. Physiol Rev 2012;92:1189234.

16. Shone CC. Clostridium botulinum neurotoxins, their structures and modes of action. In: Watson DH, editor. Natural toxicants in foods. Chichester: Ellis Harwood Ltd; 1986. p. 11-57.

17. Jankovic J. Botulinum toxin in clinical practice. J Neurol Neurosurg Psychiatry 2004;75:951-7.

18. Simpson LL. Molecular pharmacology of botulinum toxin and tetanus toxin. Annu Rev Pharmacol Toxicol 1986;26:427-53.

19. Black JD, Dolly JO. Interaction of 125I-labeled botulinum neurotoxins with nerve terminals. II. Autoradiographic evidence for its uptake into motor nerves by acceptor-mediated endocytosis. J Cell Biol 1986;103:535-44.

20. Pijning AE, Chiu J, Yeo RX, Wong JW, Hogg PJ. Identification of allosteric disulfides from labile bonds in X-ray structures. $\mathrm{R}$ Soc Open Sci 2018;5:171058.

21. Pearce LB, First ER, MacCallum RD, Gupta A. Pharmacologic characterization of botulinum toxin for basic science and medicine. Toxicon 1997;35:1373-412.

22. Brin MF, Dressler D, Aoki KR. Pharmacology of botulinum toxin therapy. In: Brin MF, Comella C, Jankovic J, editors. Dys- 
tonia: etiology, clinical feature, and treatment. Philadelphia: Lippincott Williams \& Wilkins; 2004. p. 93-108.

23. Jung GS, Cho IK, Sung HM. Submandibular gland reduction using botulinum toxin type A for a smooth jawline. Plast Reconstr Surg Glob Open 2019;7:e2192.

24. Park MY, Ahn KY, Jung DS. Botulinum toxin type A treatment for contouring of the lower face. Dermatol Surg 2003;29:47783.

25. Park MY, Ahn KY. The comparison of long-term effect of botox $(\mathrm{R})$ injection on lower face contouring after single injection and long-term repeated injections by standardized photograph analysis. J Korean Soc Plast Reconstr Surg 2009;36:654-9.

26. Park MY, Porto DA, Ahn KY. Contouring of the lower face and the lower leg and calf. In: Cohen JL, Ozog DM, Porto DA, editors. Botulinum toxins: cosmetic and clinical applications. Hoboken: John Wiley \& Sons Inc.; 2017. p. 177-90.

27. Lee JH, Lee KY, Kim JY, Son WH, Jeong JH, Gil Jeong Y, et al. Botulinum toxin injection-site selection for a smooth shoulder line: an anatomical study. Biomed Res Int 2017;2017:3092720.

28. Al-Fouzan AF, Mokeem LS, Al-Saqat RT, Alfalah MA, Alharbi MA, Al-Samary AE. Botulinum toxin for the treatment of Gummv smile. J Contemp Dent Pract 2017;18:474-8.

29. Matarasso A, Matarasso SL, Brandt FS, Bellman B. Botulinum A exotoxin for the management of platysma bands. Plast Reconstr Surg 1999;103:645-52.

30. Bi M, Sun P, Li D, Dong Z, Chen Z. Intralesional injection of botulinum toxin type A compared with intralesional injection of corticosteroid for the treatment of hypertrophic scar and keloid: a systematic review and meta-analysis. Med Sci Monit 2019;25:2950-8.

31. Kandhari R, Kaur I, Sharma D. Mesococktails and mesoproducts in aesthetic dermatology. Dermatol Ther 2020;33:e14218.

32. Childers MK. Targeting the neuromuscular junction in skeletal muscles. Am J Phys Med Rehabil 2004;83(10 Suppl):S38-44.

33. Ramirez-Castaneda J, Jankovic J, Comella C, Dashtipour K, Fernandez HH, Mari Z. Diffusion, spread, and migration of botulinum toxin. Mov Disord 2013;28:1775-83.

34. Carli L, Montecucco C, Rossetto O. Assay of diffusion of different botulinum neurotoxin type a formulations injected in the mouse leg. Muscle Nerve 2009;40:374-80.

35. Stone HF, Zhu Z, Thach TQ, Ruegg CL. Characterization of diffusion and duration of action of a new botulinum toxin type A formulation. Toxicon 2011;58:159-67.

36. Borodic GE, Ferrante R, Pearce LB, Smith K. Histologic assessment of dose-related diffusion and muscle fiber response after therapeutic botulinum A toxin injections. Mov Disord 1994;9: 31-9.

37. Restani L, Antonucci F, Gianfranceschi L, Rossi C, Rossetto O, Caleo M. Evidence for anterograde transport and transcytosis of botulinum neurotoxin A (BoNT/A). J Neurosci 2011;31:15650-9.

38. Aoki KR. Botulinum toxin: a successful therapeutic protein. Curr Med Chem 2004;11:3085-92.

39. Francisco GE, Tan H, Green M. Do botulinum toxins have a role in the management of neuropathic pain? A focused review. Am J Phys Med Rehabil 2012;91:899-909.

40. Dressler D, Benecke R. Autonomic side effects of botulinum toxin type $\mathrm{B}$ treatment of cervical dystonia and hyperhidrosis. Eur Neurol 2003;49:34-8.

41. Tintner R, Gross R, Winzer UF, Smalky KA, Jankovic J. Autonomic function after botulinum toxin type A or B: a doubleblind, randomized trial. Neurology 2005;65:765-7.

42. Huang W, Foster JA, Rogachefsky AS. Pharmacology of botulinum toxin. J Am Acad Dermatol 2000;43(2 Pt 1):249-59.

43. Kim SH, Lee SJ, Kim HJ, Lee JH, Jeong HS, Suh IS. Aging-related changes in the mid-face skin elasticity in East Asian women. Arch Craniofac Surg 2019;20:158-63. 